\title{
A systematic review and meta-analysis of the effects of clinical pathways on length of stay, hospital costs and patient outcomes Thomas Rotter* ${ }^{\dagger 1}$, Joachim Kugler ${ }^{\dagger 1}$, Rainer Koch ${ }^{\dagger 2}$, Holger Gothe ${ }^{\dagger 3}$, Sabine Twork ${ }^{\dagger 1}$, Jeroen $M$ van Oostrum ${ }^{\dagger 4}$ and Ewout W Steyerberg ${ }^{\dagger 5}$
}

\begin{abstract}
Address: ${ }^{1}$ Department of Public Health, Dresden Medical School, University of Dresden, Dresden, Germany, ${ }^{2}$ Institute of Medical Statistics, University of Dresden, Dresden, Germany, ${ }^{3}$ IGES Institute GmbH, Berlin, Germany, ${ }^{4}$ Econometric Institute, Erasmus University Rotterdam, Rotterdam, The Netherlands and ${ }^{5}$ Center for Medical Decision Making, Department of Public Health, Erasmus MC University Medical Center Rotterdam, Rotterdam, The Netherlands

Email: Thomas Rotter* - Thomas.Rotter@health-con.de; Joachim Kugler - kugler@imib.med.tu-dresden.de; Rainer Koch - koch@imib.med.tudresden.de; Holger Gothe - gt@iges.de; Sabine Twork - sabine_twork@hotmail.com; Jeroen M van Oostrum - vanoostrum@few.eur.nl; Ewout W Steyerberg - e.steyerberg@erasmusmc.nl

* Corresponding author †Equal contributors
\end{abstract}

Published: 19 December 2008

BMC Health Services Research 2008, 8:265 doi:10.1 186/1472-6963-8-265
Received: 7 July 2008

Accepted: 19 December 2008

This article is available from: http://www.biomedcentral.com/l472-6963/8/265

(C) 2008 Rotter et al; licensee BioMed Central Ltd.

This is an Open Access article distributed under the terms of the Creative Commons Attribution License (http://creativecommons.org/licenses/by/2.0), which permits unrestricted use, distribution, and reproduction in any medium, provided the original work is properly cited.

\begin{abstract}
Background: To perform a systematic review about the effect of using clinical pathways on length of stay (LOS), hospital costs and patient outcomes. To provide a framework for local healthcare organisations considering the effectiveness of clinical pathways as a patient management strategy.

Methods: As participants, we considered hospitalized children and adults of every age and indication whose treatment involved the management strategy "clinical pathways". We include only randomised controlled trials (RCT) and controlled clinical trials (CCT), not restricted by language or country of publication. Single measures of continuous and dichotomous study outcomes were extracted from each study. Separate analyses were done in order to compare effects of clinical pathways on length of stay (LOS), hospital costs and patient outcomes. A random effects meta-analysis was performed with untransformed and log transformed outcomes.
\end{abstract}

Results: In total 17 trials met inclusion criteria, representing 4,070 patients. The quality of the included studies was moderate and studies reporting economic data can be described by a very limited scope of evaluation. In general, the majority of studies reporting economic data (LOS and hospital costs) showed a positive impact. Out of 16 reporting effects on LOS, 12 found significant shortening. Furthermore, in a subgroup-analysis, clinical pathways for invasive procedures showed a stronger LOS reduction (weighted mean difference (WMD) -2.5 days versus -0.8 days)).

There was no evidence of differences in readmission to hospitals or in-hospital complications. The overall Odds Ratio (OR) for re-admission was I.I (95\% Cl: 0.57 to 2.08) and for in-hospital complications, the overall OR was 0.7 ( $95 \% \mathrm{Cl}: 0.49$ to I.0). Six studies examined costs, and four showed significantly lower costs for the pathway group. However, heterogeneity between studies reporting on LOS and cost effects was substantial.

Conclusion: As a result of the relatively small number of studies meeting inclusion criteria, this evidence base is not conclusive enough to provide a replicable framework for all pathway strategies. Considering the clinical areas for implementation, clinical pathways seem to be effective especially for invasive care. When implementing clinical pathways, the decision makers need to consider the benefits and costs under different circumstances (e.g. market forces). 


\section{Background}

Clinical pathways represent a form of "cookbook medicine" that many perceive as an appropriate tool that contributes to quality management, cost-cutting and patient satisfaction.

For the aim of this review, clinical pathways are defined as complex interventions consisting of a number of components based on the best available evidence and guidelines for specific conditions [1]. A clinical pathway defines the sequencing and timing of health interventions and should be developed through the collaborative effort of physicians, nurses, pharmacists, and other associated health professionals [2]. Clinical pathways aim to minimize delays and maximize resource utilization and quality of care [1]. They are also referred to as "integrated care pathways", "critical pathways", "care plans", "care paths", "care maps" and "care protocols".

The effectiveness of clinical pathways is under debate. However, especially in the US, up to 80 percent of hospitals already use clinical pathways for at least some indications [3]. A number of primary studies considered the effectiveness of clinical pathways, but results are inconsistent and suffer from various biases [4-7]. Only one systematic review has been performed, specifically for stroke patients [8]. Narrative reviews are more common, which often rely on "expert opinions" [9-11].

We perform a systematic review and a random effects meta-analysis to assess whether clinical pathways improved the outcome measures "length of stay (LOS)", "hospital costs" and "quality of care" when compared to standard care. By performing a systematic review and meta-analysis we are able to present the available evidence in a substantiated and concise way, in order to provide a framework for local healthcare organisations considering the effectiveness of clinical pathways.

\section{Methods}

We followed the methods of the Cochrane Collaboration [12] with some modifications, mainly concerning presentation of meta-analytic results.

\section{Study selection criteria}

As potential patient samples we considered hospitalized children and adults of every age and indication, whose treatment involved the management strategy "clinical pathways". Given the problem that there are variations in the terminology used in the current research [13], we defined minimum "inclusion criteria" for meeting our clinical pathway definition (see Table 1). Based on our definition (see background), we developed a pre-specified, three operational pathway criteria as follows: 1) multidisciplinary (two or multiple clinical professions involved), 2) protocol or algorithm based (i.e. structured care plan/treatment-protocol or algorithm) and finally, 3) evidence based (pathway components were minimally based on one RCT or best practice guidelines). Every pathway characteristic could be met as (1) "yes" criterion; (2) "not sure" because of poor reporting and the failure to contact the principal author or (3) "criterion not met." If one or more pathway criteria selected is not met, then we excluded the study.

Please note, additional information relating to the included studies that matched these requirements or differ from each other, are given in the results section of this review.

The setting definition covered the whole range of services offered by the clinical (out- and in-patient) as well as in the in-patient rehabilitation sector. We only gathered robust evidence and limited our study selection to randomised controlled trials (RCT) and controlled clinical trials (CCT) including methodological quality criteria (please see "quality assessment and data analyses").

We considered every objective economic and patient outcome for inclusion. We pre-defined (1) in-hospital complications as a secondary disease or adverse medical occurrence during hospitalization [14] and (2) we defined re-hospitalization as a readmission within a specified follow up period of an index admission.

\section{Data sources and search strategy}

We performed specialised searches of the Medline database (1966-2006), Embase (1980-2006), Cinahl (19822006), Global Health (1973-2006), and the specialised Cochrane register (including NHS EED and HTA Database; last update: 13.11.06), not restricted by language or country. We used free text words (tw), medical subject headings (MeSH terms -/-) or exploded MeSH terms for our MEDLINE literature search. This controlled vocabulary was adapted (as much as possible) to the indexation (thesaurus) of all other databases included in this review. We demonstrate our "clinical pathway search strategy" with the MEDLINE inquiry (Table 2).

Furthermore, we employed citation tracking, which examines included studies and previous reviews and contacted investigators to identify any study missed by the electronic searches.

\section{Quality assessment and data analysis}

For quality of studies (see additional File 1), we adhered to the Effective Organisation of Care Group (EPOC) module [15] and defined three risk classes: Class I (low risk of bias), Class II (moderate risk of bias) and Class III (high risk of bias). Two reviewers independently assessed and 
Table I: Pathway characteristics and quality outcome measures of studies included

\begin{tabular}{|c|c|c|c|c|c|c|}
\hline Pathway & $\begin{array}{l}\text { Charac- } \\
\text { teristics }\end{array}$ & & & Quality Measure & Pathway $[\mathrm{n} / \mathrm{N}]$ & Control $[\mathrm{n} / \mathrm{N}]$ \\
\hline Study-ID & $\underset{\text { multi- }}{\text { disciplinary }}$ & evidence-based & $\begin{array}{l}\text { protocol/ } \\
\text { algorithm } \\
\text { based }\end{array}$ & & $\begin{array}{l}\text { Counts and rates } \\
\text { are presented in } \\
\text { natural units and } \\
\text { as percentages as } \\
\text { far as reported }\end{array}$ & $\begin{array}{l}\mathrm{N}=\text { numer of } \\
\text { participants } n= \\
\text { number of } \\
\text { events }(\%)= \\
\text { percentage }\end{array}$ \\
\hline \multicolumn{7}{|l|}{ Invasive Care } \\
\hline Grines, CL & $x$ & $x$ & $x$ & $\begin{array}{l}\text { In-hospital } \\
\text { complications }\end{array}$ & $20 / 237$ (8.4\%) & $20 / 234$ (8.5\%) N.S. \\
\hline 1998 & & & & $\begin{array}{l}\text { Re-hospitalisation } \\
\text { (6 months) }\end{array}$ & 10/237 (4.2\%) & 9/234 (3.9\%) N.S. \\
\hline Swanson, CE & $x$ & Not & $x$ & Hospital mortality & $2 / 38(5.2 \%)$ & $2 / 33(6.1 \%)$ N.S. \\
\hline 1998 & & sure & & $\begin{array}{l}\text { Mean Modified } \\
\text { Barthel Index }\end{array}$ & 92.8 & $85.6(p<0.05)$ \\
\hline Dowsey, MM & $\mathrm{x}$ & $\mathrm{x}$ & $x$ & $\begin{array}{l}\text { In-hospital } \\
\text { complications }\end{array}$ & $10 / 92(10.8 \%)$ & $\begin{array}{l}20 / 71 \\
(28.1 \%)(p<0.05)\end{array}$ \\
\hline 1999 & & & & $\begin{array}{l}\text { Re-hospitalisation } \\
\text { ( } 3 \text { months) }\end{array}$ & $1 / 92(1.1 \%)$ & 0/7I (0\%) N.S. \\
\hline Choong, PF & $x$ & $x$ & $x$ & $\begin{array}{l}\text { In-hospital } \\
\text { complications }\end{array}$ & $10 / 55(18.2 \%)$ & I 4/56 (25.0\%) N.S. \\
\hline 2000 & & & & $\begin{array}{l}\text { Re-hospitalisation } \\
\text { ( } 28 \text { days) }\end{array}$ & $2 / 55(3.6 \%)$ & 6/56 (10.7\%) N.S. \\
\hline Aizawa, $\mathrm{T}$ & $\mathrm{x}$ & $\mathrm{x}$ & $x$ & $\begin{array}{l}\text { In-hospital } \\
\text { complications }\end{array}$ & $1 / 32(3.1 \%)$ & 2/37 (5.4\%) N.S. \\
\hline 2002 & & & & $\begin{array}{l}\text { Re-hospitalisation } \\
\text { (6 months) }\end{array}$ & $1 / 32(3.1 \%)$ & $0 / 37(0 \%)$ N.S. \\
\hline Kiyama, T & $x$ & $x$ & $x$ & $\begin{array}{l}\text { In-hospital } \\
\text { complications }\end{array}$ & $3 / 47(6.4 \%)$ & 5/38 (I3.2\%) N.S. \\
\hline \multicolumn{7}{|l|}{2003} \\
\hline Hirao, M & $x$ & $x$ & $x$ & $\begin{array}{l}\text { In-hospital } \\
\text { complications }\end{array}$ & $19 / 53(35.8 \%)$ & $17 / 50$ (34.0\%) N.S. \\
\hline 2005 & & & & $\begin{array}{l}\text { Re-hospitalisation } \\
\text { (6 months) }\end{array}$ & $0 / 53(0 \%)$ & $0 / 50(0 \%)$ \\
\hline \multicolumn{7}{|l|}{$\begin{array}{l}\text { Non-Invasive } \\
\text { Care }\end{array}$} \\
\hline Falconer, JA & $\mathrm{x}$ & Not & $x$ & $\begin{array}{l}\text { Mortality } \\
\text { (12 months) }\end{array}$ & N.S. & N.S. \\
\hline \multirow[t]{3}{*}{1993} & & Sure & & $\begin{array}{l}\text { Re-hospitalisation } \\
\text { (12 months) }\end{array}$ & N.S. & N.S. \\
\hline & & & & $\begin{array}{l}\text { Cognitive and } \\
\text { functional scores } \\
(0-100)\end{array}$ & N.S. & N.S. \\
\hline & & & & Patient satisfaction & 7.7 (SD 2.6) & $\begin{array}{l}8.8 \\
(S D \text { I.7) }(p<0.05)\end{array}$ \\
\hline Gomez, MA & $\mathrm{X}$ & $X$ & $\mathrm{X}$ & $\begin{array}{l}\text { Complete and } \\
\text { graded exercise } \\
\text { test }\end{array}$ & $44 / 50(88.0 \%)$ & I5/50 (30.0\%) \\
\hline \multicolumn{7}{|l|}{1996} \\
\hline Roberts, RR & $x$ & $x$ & $x$ & $\begin{array}{l}\text { Hospitalised } \\
\text { patients as \% }\end{array}$ & $(45.1 \%)$ & $(100 \%)$ \\
\hline 1997 & & & & $\begin{array}{l}\text { Re-hospitalisation } \\
\text { (8 weeks) }\end{array}$ & $5 / 82(6.1 \%)$ & $4 / 83(4.8 \%)$ N.S. \\
\hline Johnson, KB 2000 & $x$ & $x$ & $\mathrm{X}$ & $\begin{array}{l}\text { Unscheduled clinic } \\
\text { visits; no hospital } \\
\text { re-admission } \\
\text { ( } 2 \text { weeks) }\end{array}$ & $0 / 55(0 \%)$ & $2 / 55(3.6 \%)$ \\
\hline Kollef, HM & $x$ & $x$ & $\mathrm{X}$ & $\begin{array}{l}\text { In-hospital } \\
\text { complications }\end{array}$ & $9 / 239(3.8 \%)$ & I3/250 (5.2\%) N.S. \\
\hline 2000 & & & & Hospital mortality & $5 / 239(2.1 \%)$ & $8 / 250$ (3.2\%) N.S. \\
\hline
\end{tabular}


Table I: Pathway characteristics and quality outcome measures of studies included (Continued)

\begin{tabular}{|c|c|c|c|c|c|c|}
\hline \multirow[t]{4}{*}{ Marrie, TJ 2000} & $\mathrm{X}$ & $\mathrm{X}$ & $\mathrm{X}$ & $\begin{array}{l}\text { Absolute difference } \\
\text { in rates (ARR) } \\
\text { between pathway } \\
\text { and control }\end{array}$ & & $\begin{array}{l}\text { I-sided 95\% Cl } \\
\text { upper limit: }\end{array}$ \\
\hline & & & & $\begin{array}{l}\text { In-hospital } \\
\text { complications }\end{array}$ & $(0.6 \%)$ & (4.6\%) N.S. \\
\hline & & & & $\begin{array}{l}\text { Re-hospitalisation } \\
\text { (6 weeks) }\end{array}$ & $(0.7 \%)$ & (3.6\%) N.S. \\
\hline & & & & Mortality ( 6 weeks) & $(-0.1 \%)$ & $(2.5 \%)$ N.S. \\
\hline Sulch, D & $x$ & $x$ & $x$ & $\begin{array}{l}\text { Median Barthel } \\
\text { Index Score } \\
\text { ( } 26 \text { weeks) }\end{array}$ & 17 & I 7 N.S. \\
\hline 1999 & & & & $\begin{array}{l}\text { Mortality } \\
\text { (26 weeks) }\end{array}$ & $10 / 76(13.2 \%)$ & 6/76 (7.9\%) N.S. \\
\hline $\mathrm{Kim}, \mathrm{MH}$ & $x$ & $x$ & $x$ & $\begin{array}{l}\text { Complications until } \\
\text { follow-up ( } 27 \text { days) }\end{array}$ & I/9 (II.1\%) & I/9 (II.1\%) N.S. \\
\hline 2002 & & & & $\begin{array}{l}\text { Re-hospitalisation } \\
\text { ( } 27 \text { days) }\end{array}$ & $2 / 9(22.2 \%)$ & $0 / 9(0 \%)$ N.S. \\
\hline \multirow[t]{2}{*}{ Chen, SH 2004} & $x$ & $x$ & $x$ & $\begin{array}{l}\text { Emergency room } \\
\text { usage (not } \\
\text { comparable with } \\
\text { in-hospital } \\
\text { complications) }\end{array}$ & $3 / 20(15.0 \%)$ & $\begin{array}{l}13 / 22 \\
(59.1 \%)(p<0.05)\end{array}$ \\
\hline & & & & $\begin{array}{l}\text { Re-hospitalisation } \\
\text { ( } 3 \text { months) }\end{array}$ & N.S. & N.S. \\
\hline Usui, K 2004 & $x$ & $x$ & $x$ & Not reported & & \\
\hline
\end{tabular}

Legend: Every pathway characteristic could be met as (I) "yes" criterion; (2) "not sure" because of poor reporting and the failure to contact the principal author or (3) "criterion not met." If one or more pathway criteria selected is/are not met, then we excluded the study. Due to poor reporting some quality measures are presented only as percentages or mean scores with or without associated standard deviations (SD). Some quality measures were only reported as statistical significant (i.e. $\mathrm{p}<0.05$ ) or not significant (N.S.) and any other data were missing.

Table 2: Clinical pathway search strategy Ovid Medline: 1966 to November Week 22006

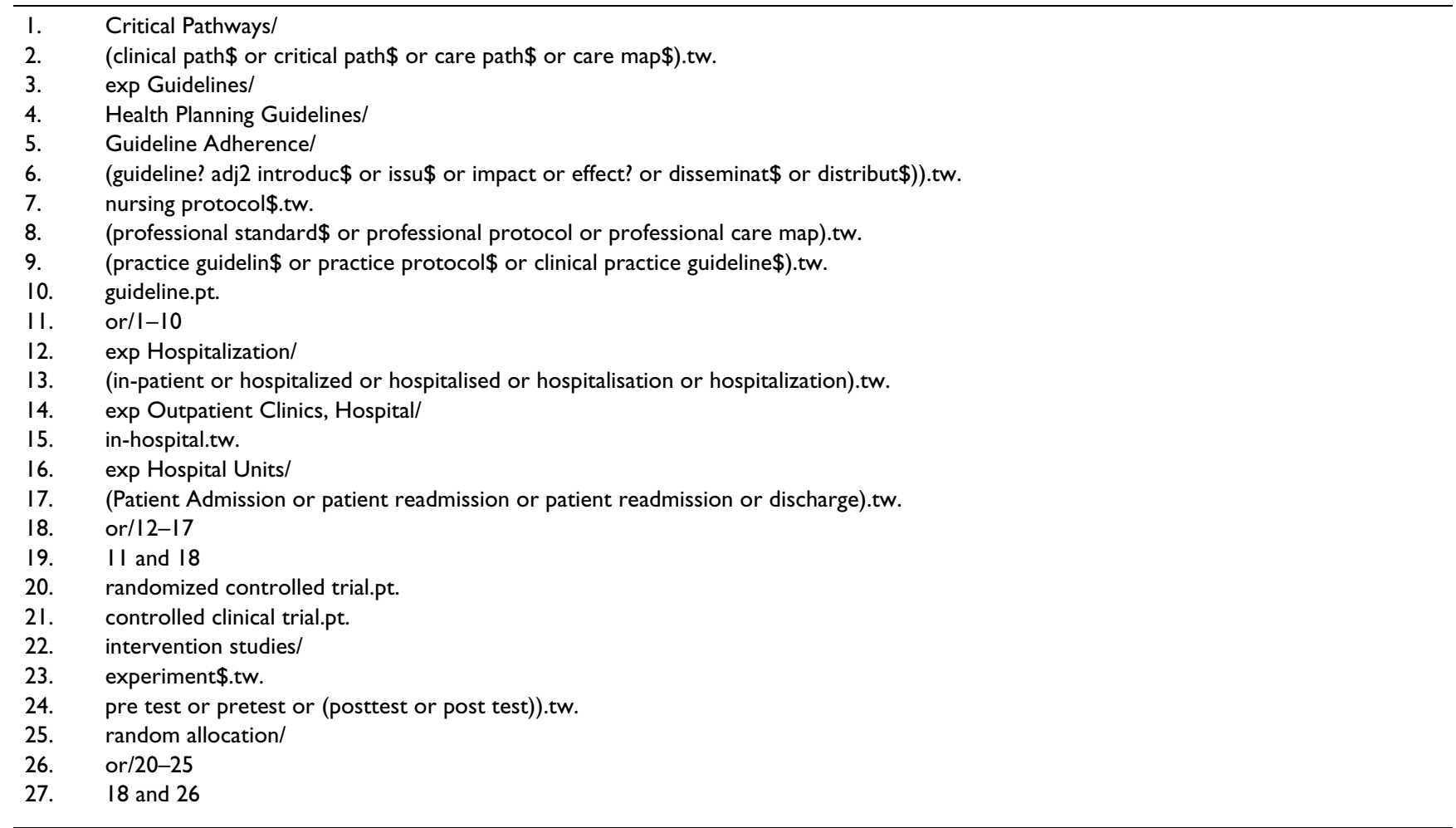


abstracted data, on the intervention criteria, study characteristics and methodological quality. Any disagreement was discussed with a third reviewer. Studies with a high risk of bias were excluded from the review after documentation. If a primary study did not provide information about the standard deviation, we used the approximative or direct algebraic connection between the stated confidence intervals, or p-values, and the standard deviation and calculated the inverse transformation to the individual or pooled standard deviation [12]. Prior to the actual statistical pooling of the singular effects, an adjustment of the data regarding costs due to inflation and price adjustment (OECD Health Care Price Index) was carried out [16]. We chose the US Dollar (USD) as the basic currency. The year 2000 was chosen as a representative year for inflation and price adjustments or exchange rates.

We used Review Manager (RevMan) of the Cochrane Collaboration to calculate a pooled effect estimate, called weighted mean difference (WMD) [17]. We used a random effects model since this model estimates the effect with consideration to the variance between studies, rather than ignoring heterogeneity by employing a fixed effect model. The effect sizes were generated using a model fitting inverse variance weights [17].

\section{Heterogeneity and meta-analysis}

Despite the expected clinical heterogeneity (clinical variability of the included pathway interventions) within the review, it is important to assess the comparability of the results from individual studies. A useful statistic for quantifying inconsistency is $I^{2}=[(\mathrm{Q} d f) / Q] \times 100 \%$, where $Q$ is the chi-squared statistic and $\mathrm{df}$ is its degrees of freedom [12]. This quantifies the total variance explained by the heterogeneity as a percentage. We considered an overall test-value greater than $60 \%$ to serve as evidence of substantial heterogeneity of a magnitude were statistical pooling is not appropriate.

\section{Sensitivity analysis}

In a sensitivity analysis, both fixed effects and random effects models were employed to determine the causes of heterogeneity and test the confidence that can be placed in both estimates. Only robust estimations of the pooled effects with similar results in fixed effects and random effects models are included in the meta-analysis and discussed. Furthermore, sensitivity analyses were performed to test whether the effect size varied by the countries where the study was carried out (adjusting for market forces) and the year of publication, adjusting for temporal trends.

\section{Subgroup analysis}

We decided previously to perform a subgroup analysis of invasive versus non-invasive clinical pathways, where- upon the distinction between invasive or non-invasive interventions refers to the nature of patient management guided by clinical pathways (i.e. clinical pathways for gastrectomy; transurethral resection of the prostate; hip and knee arthroplasty; percutaneous transluminal coronary angioplasty; etc. versus clinical pathways for asthma care; stroke; pneumonia; etc.). According to theories on health economics, invasive procedures can be standardized more easily than treatment strategies in conservative sectors due to the lower treatment variance [18].

\section{Assessing publication bias}

We used a funnel plot analysis to assess publication bias, i.e. the bias caused by a lower likelihood of publication for non-significant studies. The funnel plot is a scatterplot with the $\mathrm{x}$-axis representing the effects estimated from the primary studies, and the $y$-axis representing a measure of the sample size in each study (SE; standard error of the mean) [19]. If publication bias is absent, the diagram shows an inverted symmetrical funnel.

However, given that publication bias poses a threat to the validity of this meta-analysis and the graphical method is subjective in nature, we also applied a statistical approach, often called fail-safe $\mathrm{N}$ test.

This test provides an estimate of the number of unpublished or in this context called file-draw studies (Nfs) having an average of no effect that is necessary in the analysis for reducing the pooled effect size from significant to nonsignificant [20]. Using a critical d level (d crit) of -0.20, the estimate of unpublished studies was calculated with

$$
\mathrm{Nfs}=(\text { Ntotal }(\text { mean } \mathrm{d}-\mathrm{d} \text { crit })) / \mathrm{d} \text { crit; }
$$

where Ntotal is the total number of studies included in the Meta-Analysis and d is the overall pooled effect (WMD).

\section{Secondary analyses}

The distribution of the length of stay is limited downwards in a natural way (because of the minimum value is always 0 ), whereas upwards the values can scatter significantly. According to logarithmic transformation, values with this characteristic can have an approximately normal distribution [21]. In the additional File 2 we detailed the formulas used for log transformation. Data analyses were performed using SPSS version 15.0 [22].

\section{Results}

\section{Search strategy and intervention characteristics}

The specialised search strategy led to the initial selection of 2,386 studies, whereas only 17 matched our methodical requirements (see Figure 1 \&2). For the first stage of the study assessment, we scanned all of the 2,386 titles and abstracts for inclusion, the remaining 256 possibly 


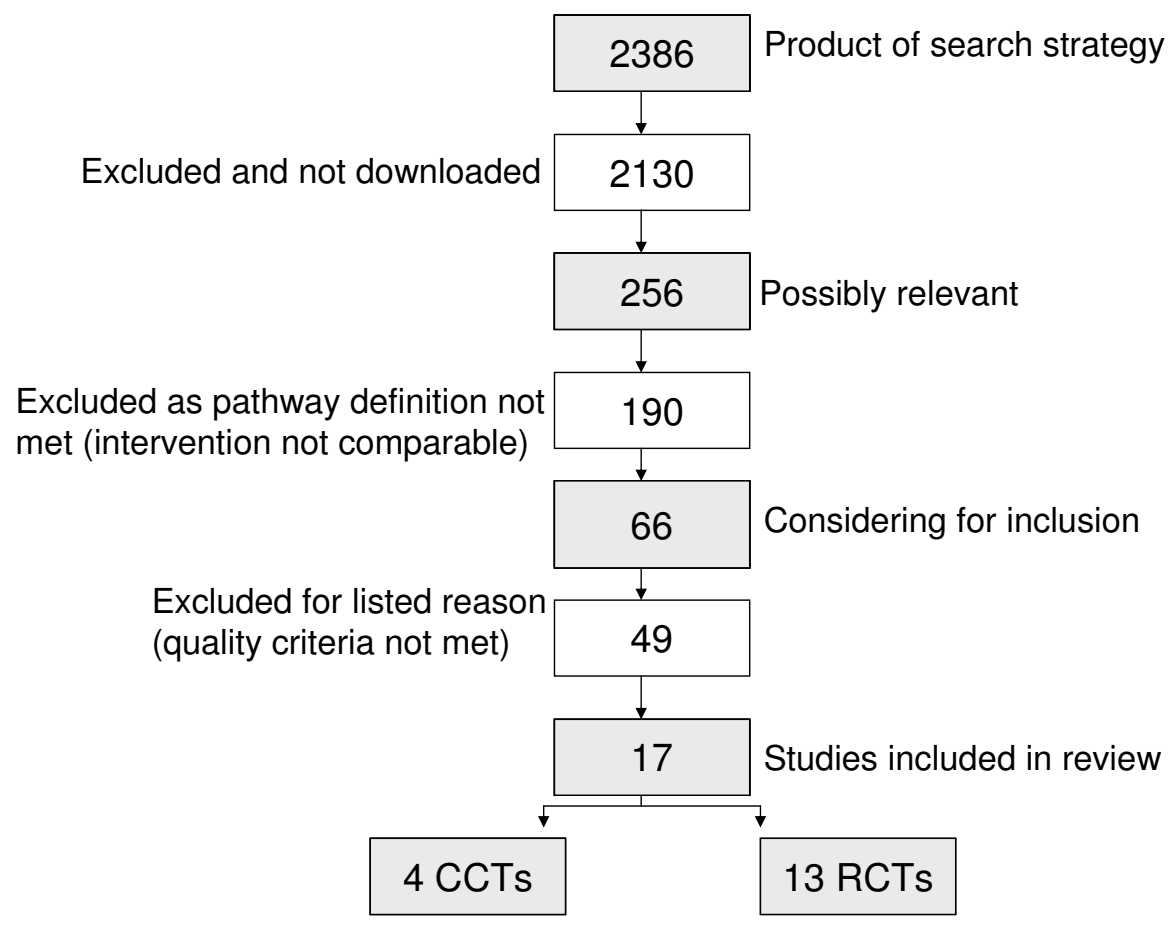

Figure I

Identification of relevant studies/trail flow. [see attached file I] (PDF Format).

relevant studies were retrieved as full text articles. Based on the full text assessment, we excluded 190 studies out of 256 because they failed to meet our pathway definition. The majority of the excluded studies failed to meet the multidisciplinary pathway criterion, i.e. it was a therapy guideline issued by a medical association or it was a uniprofessional nursing care plan. Others did not meet the "algorithm or protocol based" criterion because there was no structure and detailed care plan. For example a poster with issued guidelines was posted in the Emergency Department.

Evidence for meeting the minimal criterion "pathway content is minimally based on one RCT" was reported in 15 studies out of 17 , which were included in the review. The study from Falconer et al. and Swanson at al. met the evidence criterion, "not sure" because we failed in contacting the principal investigators $[23,24]$.

\section{Intervention characteristics}

The reported pathway strategies can be described as complex pathway interventions versus a "non-intervention" control group or often poorly, described as "usual or traditional care" group. Most of the experimental interventions were combined with other types of interventions like audit and feedback, educational meetings, and reminders. For 8 out of the 17 (47\%) included studies, it was clear that the structured care plan was combined with a "clinical diagnostic or assessment protocol"[23,25-31].

The evidence base for two (12\%) pathway interventions was "not sure," whereas the remaining 15 interventions were minimally based on one randomized study or good evidence. The reported purpose of the pathway strategies was appropriate management or cost containment.

The hospital setting was in two studies a multi-center study comprising a range of hospitals included in the investigation $[26,30]$. From the 15 remaining single center studies, 8 studies (53\%) were carried out in a university (teaching) hospital setting [24,27-29,32-35] and 7 studies $(46 \%)$ in a non-university hospital setting [23,25,31,36-39].

Details of the intervention characteristics are given in Table 3.

\section{Quality assessment}

To summarize, we examined the design and study quality of 66 studies, excluding 49 out of the 66 studies because of the high risk of bias (see trail flow, Figure 1). Characteristics (reason for exclusion: see additional File 3) of the 49 excluded studies (references to excluded studies: see additional File 4) are given in detail. 


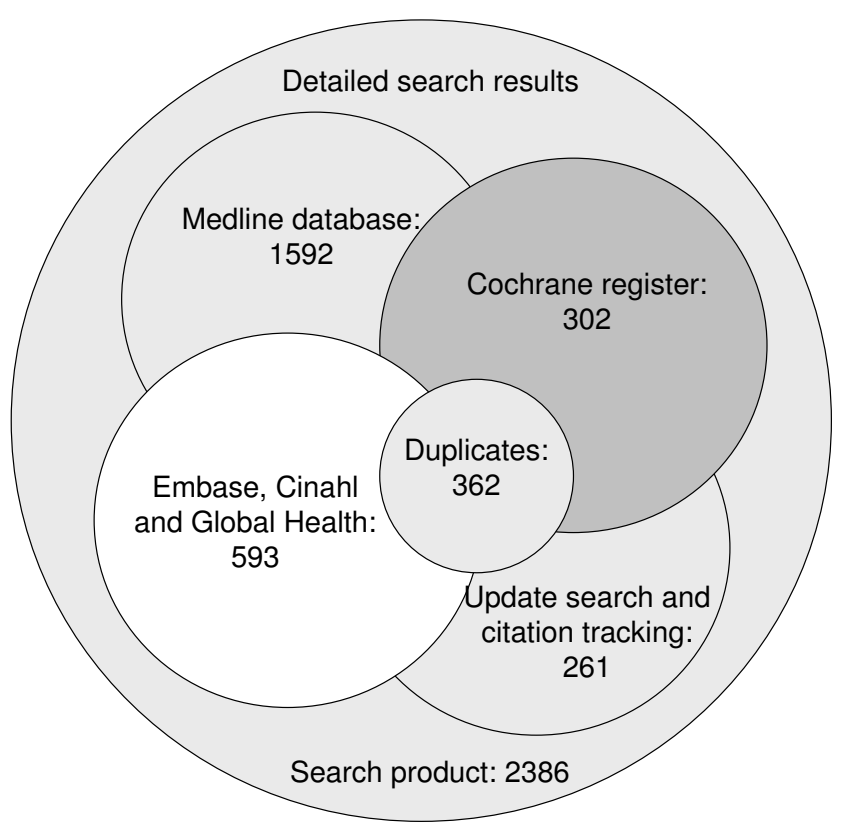

Figure 2

Electronic search results. [see attached file 2] (PDF Format).
The patient was randomized to the experimental or control group in 12 out of 13 (92\%) RCTs. The randomization process was clear in all such studies and justified by the authors. Referring to the individually randomized and single-center studies, the assessment of protection against contamination of the control professionals remained unclear due to poor reporting. None of the investigators reported protection against contamination (communication between experimental and control professionals) and it is possible that control subjects received the intervention. Only the investigation from Marie et al. used a robust cluster randomized design, with 19 hospitals as unit of allocation [30]. To avoid "unit of analysis error," we conducted the meta-analysis at the same level as the allocation (19 cluster-hospitals $=19$ patients ).

Poor reporting also lead to difficulties in determining the assessment of the power calculations. For instance, sample-size calculation was unclear for over $60 \%$ of the included studies; hence the study sample may not have been sufficiently large. Another problem, due to poor reporting was the selection of comparators. The choice of the comparator (i.e. the control and intervention units were located either in the main building or the east building of the participating hospital) was stated and justified by the authors of the 17 primary studies. However, a clear description of what was meant by traditional care or usual

Table 3: Characteristics of studies included

\begin{tabular}{|c|c|c|c|c|c|}
\hline Study-ID & Study Quality & Country & Sample Size [N] & Mean Age [Years] & Diagnosis/Intervention \\
\hline \multicolumn{6}{|l|}{ Invasive Care } \\
\hline Grines, CL 1998 & Class I & USA* & 471 & 56 & Primary Angioplasty in Myocardial Infarction \\
\hline Swanson, CE 1998 & Class II & Australia & 67 & 55 & Femoral Fractures \\
\hline Dowsey, MM 1999 & Class II & Australia & 163 & 66 & Hip and Knee Arthroplasty \\
\hline Choong, PF 2000 & Class II & Australia & 111 & 81 & Fractured Neck of Femur \\
\hline Aizawa, T 2002 & Class II & Japan & 69 & 71 & Transurethral Resection of the Prostate \\
\hline Kiyama, T 2003 & Class II & Japan & 85 & 63 & Gastrectomy \\
\hline Hirao, M 2005 & Class II & Japan & 103 & 61 & Gastrectomy \\
\hline \multicolumn{6}{|c|}{ Non-Invasive Care } \\
\hline Falconer, JA 1993 & Class II & USA* & 121 & 68 & Stroke Rehabilitation \\
\hline Gomez, MA 1996 & Class I & USA* & 100 & 52 & Myocardial Ischemia \\
\hline Roberts, RR 1997 & Class II & USA* & 165 & 48 & Chest Pain \\
\hline Johnson, KB 2000 & Class II & USA* & 110 & 7 & Paediatric Asthma \\
\hline Kollef, HM 2000 & Class II & USA* & 489 & 60 & Respiratory Care \\
\hline Marrie, TJ 2000 & Class I & USA* & $19 * * *$ & 64 & Community-Acquired Pneumonia \\
\hline Sulch, D 2000 & Class II & UK** & 152 & 75 & Stroke Rehabilitation \\
\hline Kim, MH 2002 & Class II & USA* & 18 & 48 & Artrial Fibrillation \\
\hline Chen, SH 2004 & Class II & Taiwan & 42 & 8 & Paediatric Asthma \\
\hline Usui, K 2004 & Class II & Japan & 61 & 48 & Community-Acquired Pneumonia \\
\hline
\end{tabular}

Note: USA* = United States of America; UK** = United Kingdom; 19*** = (19) hospitals at random (I743 Patients) 
care (control group) would have helped in assessing the relevance of the study to other settings.

Primary studies reporting economic data, can be described by a very limited scope of evaluation, focusing on direct hospital LOS and costs effects, rather than on a full economic evaluation [16]. In Table 3 the quality assessment and characteristics of the 17 studies included in the review and meta-analysis are shown in detail.

\section{Effects on LOS}

Out of the 16 studies (12 randomized and four non-randomized studies representing a study population of 4,028 patients) examining the effect of clinical pathways on the length of stay, 12 showed significant effects [23,24,2639]. However, heterogeneity between studies reporting on LOS was substantial $\left(\mathrm{I}^{2}=80 \%\right)$ and may refer to both the statistical inconsistency as well as to the varying clinical pathway interventions that were included. As a result, the estimation of an overall pooled effect is not appropriate and in Figure 3, the differences from the individual studies in LOS are depicted together with the corresponding confidence intervals without totals. The reported LOS in Kiyama 2003 was calculated from the day of surgery to the day of discharge [39]. All other studies included in this analysis considered the total LOS.

\section{Effects on patient outcomes}

Out of 17 trials reporting effects on quality outcome measures (see Table 1); six measures were comparable in terms of re-hospitalisation and seven in terms of in-hospital complications [23-35,37-39]. In total, nine primary studies were included in the Meta-analysis (representing a study population of 1,674 patients), examining the effect of clinical pathways on quality patient outcomes. The pooled Odds Ratio (OR) for re-admission was 1.1 (95\% CI: 0.57 to 2.08 ) and for in-hospital complications the overall OR was 0.7 (95\% CI: 0.49 to 1.0). Statistical heterogeneity was not present among the studies and there was no evidence of difference in readmission to hospitals or in-hospital complications. The effects of clinical pathways on clinical outcomes in the individual studies are depicted together with the pooled OR (see Figure 4 \&5). There was clinical variance in the range of follow-up periods that were used by the investigators measuring re-hospitalization (follow up periods ranged from 27 days to 6 month, see Table 1) as well as the investigators used varying definitions of the term in-hospital complications (included in-hospital complications were cardiac events, infections, thrombosis, re-operation, sepsis and empyema). Obviously, this implies that any time element in the patient outcome data is lost through this approach and it was not possible to compute a series of dichotomous outcomes, i.e. at least one event during the first year of follow up.

\section{Effects on hospital costs}

Six of the included studies (five randomized and one nonrandomized), representing a sample of 1328 treated patients, reported on cost effects [26,28,31,33,34,39]. Four out of the five randomized studies found significantly lower hospitalisation costs for pathway groups. The statistical heterogeneity was substantial $\left(\mathrm{I}^{2}=88 \%\right)$ and compromised the estimation of a pooled effect. Additionally, we also observed a considerable methodological variation which refers to the different methods of cost calculation used by the investigators. Some investigators used a full cost approach (fix and variable costs included),

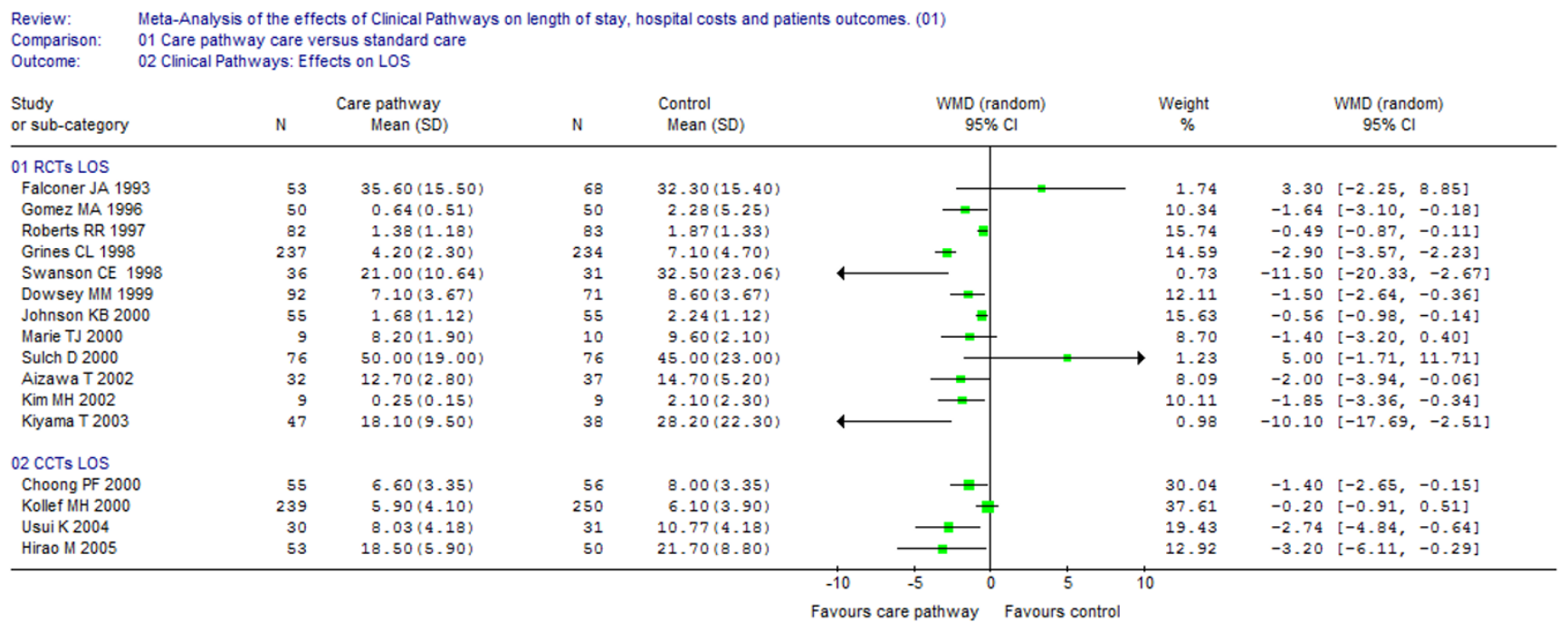

Figure 3

Effects on LOS. [see attached file 3] (BMP Format). 


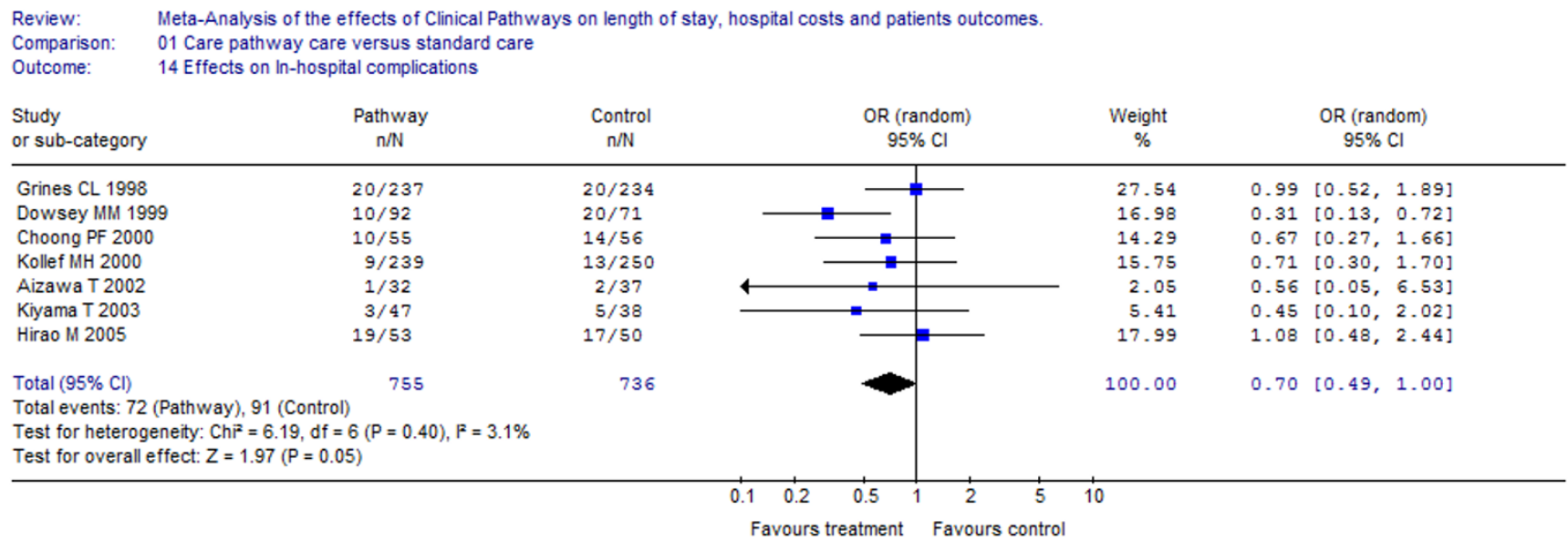

Figure 4

Effects on in-hospital complications. [see attached file 4] (BMP Format).

whereas others calculated only direct hospital costs. Table 4 describes the costs differences in detail.

\section{Subgroup analysis: invasive versus non-invasive clinical pathways}

Five of the randomized studies, and two further non-randomized studies assessed the LOS effects of surgical or minimally invasive interventions [24,26,27,29,35,37,39]. The pooled effect for all invasive primary studies was -2.5 days (95\% CI: -3.53 to -1.41). The differences in LOS in the individual studies are depicted together with the total effect per study type (RCTs versus CCTs, Figure 6). The statistical pooling of the subgroup of surgical pathway interventions is characterized by a considerable overall testvalue for statistical inconsistency $\left(\mathrm{I}^{2}=60.9 \%\right)$ which also reflects the clinical heterogeneity of the surgical pathway interventions included in this comparison.
The subgroup of the conservative pathway indications $[23,28,30-34,36,38]$ had a reduction of LOS of approximately one day (WMD $-0.75 ; 95 \% \mathrm{CI}:-1.23$ to -0.27 , Figure 7).

\section{Sensitivity analyses}

The LOS effects were robust in terms of the sensitivity analysis concerning the different statistical calculation models (fixed versus random effects model) and the Year of publication, adjusting for temporal trends. However, we observed a trend toward greater reported LOS effects from Japanese studies with a reduction of approximately three days (WMD - 2.7), followed by studies carried out in Australia (WMD - 1.5), Canada (WMD - 1.4) and the USA (WMD - 0.8). Subsequently, we tested the hypotheses, that different market forces (reported effect sizes per country) are possibly confounding the conclusions of these review and meta-analysis. After exclusion (stepwise/

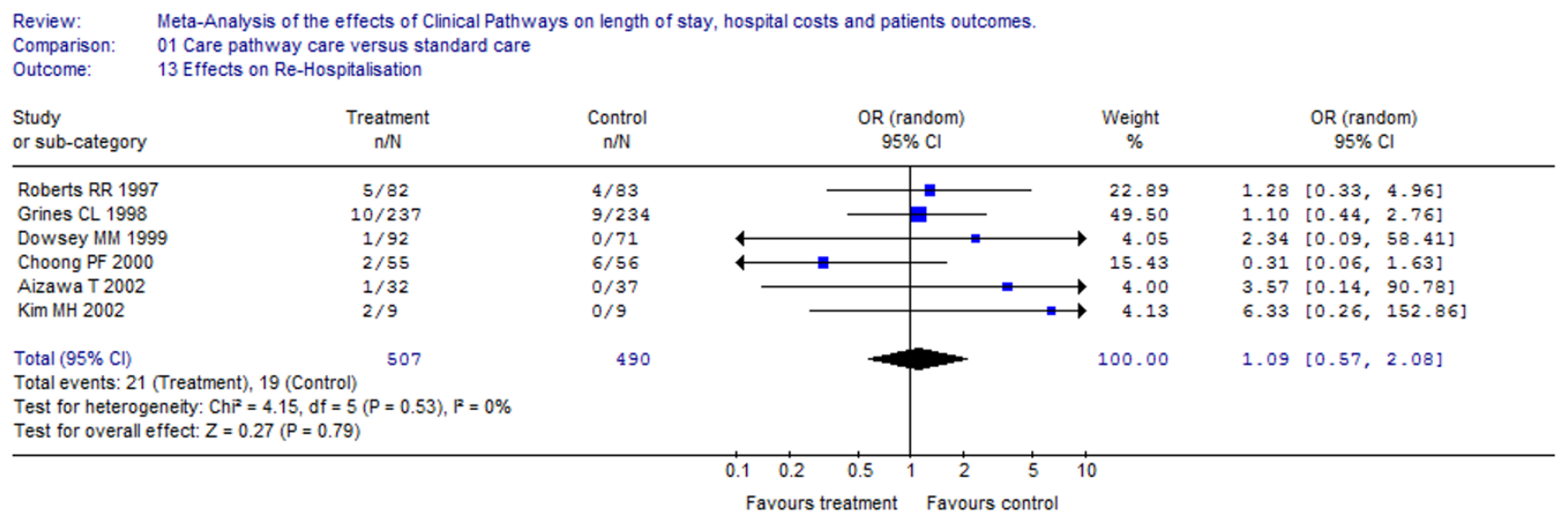

Figure 5

Effects on re-hospitalisation. [see attached file 5] (BMP Format). 
Table 4: Cost data, standardized to the year 2000

\begin{tabular}{lllllll}
\hline Study ID & Country & Currency & Experiment & SD & Control & SD \\
\hline Kiyama, T 2003 & Japan & US\$ & $\$ 14013$ & $\$ 2634$ & $\$ 18020$ & $\$ 7332$ \\
Kim, MH 2002 & USA* & US\$ & $\$ 879$ & $\$ 394$ & $\$ 1706$ & $\$ 1512$ \\
Kollef, HM 2000 & USA & US\$ & $\$ 922$ & $\$ 1614$ & $\$ 1120$ & $\$ 1430$ \\
Grines, CL 1998 & USA & US\$ & $\$ 11430$ & $\$ 6257$ & $\$ 13733$ & $\$ 7249$ \\
Roberts, RR 1997 & USA & US\$ & $\$ 1877$ & $\$ 1243$ & $\$ 2574$ & $\$ 999$ \\
Gomez, MA 1996 & USA* & US\$ & $\$ 1535$ & $\$ 1985$ & $\$ 6768$ & $\$ 17359$ \\
\hline
\end{tabular}

Note: USA* = United States of America

iterative and all of the primary Japanese studies) of the subgroup of Japanese studies, the (calculative) overall LOS effect remained robust and statistically significant, but tended to be smaller (WMD - 1.2; subgroup "Japanese studies excluded" versus WMD - 1.5; subgroup "all primary studies" included). This applies also to the subgroup Analysis "Invasive versus non-invasive LOS effects", after exclusion of the subgroup of Japanese studies (WMD -0.6 conservative versus -2.2 invasive).

In addition, the overall odds ratios (OR) for re-admission and in-hospital complications were robust in all terms of the sensitivity analysis, indicating reliable pooled results.

\section{Publication bias and other sources of systematic error}

The funnel plot showed a relatively symmetric distribution (Figure 8), but the point cloud does not have a distinctive funnel form. The deficient funnel form of the funnel plot can also be due to the relatively high heterogeneity with respect to the different pathway indications of the primary studies included in these review (crossindicational methodology of the primary studies). Fur- thermore, the number of studies was relatively small. However, given that publication bias may still exist, the statistical fail-safe $\mathrm{N}$ objectively helps to quantify. The calculation about the number of file-drawer studies showed that 101 non-significant studies would have to exist to reduce the (calculative) overall effect size of (WMD) 1.47 to a mean effect size of -0.2 . These results indicate that unpublished research is unlikely to threaten the validity of the original meta-analysis.

\section{Secondary analyses}

The graphic distribution of the original and the logarithmical (natural logarithm LN) LOS data clearly indicates that there is a significant deviation from the normal distribution (Figure 9a \&9b). The heterogeneity between studies was not substantially lowered by the log transformation.

The LOS reduction was estimated as 25 percent (95\% CI: $-36 \%$ to $-14 \%$ ). For example, with the average length of stay of 7 days, the estimated LOS effect was approximately -1.7 days ( 7 days * $-0.25=-1.75$ days LOS reduction). Fur-

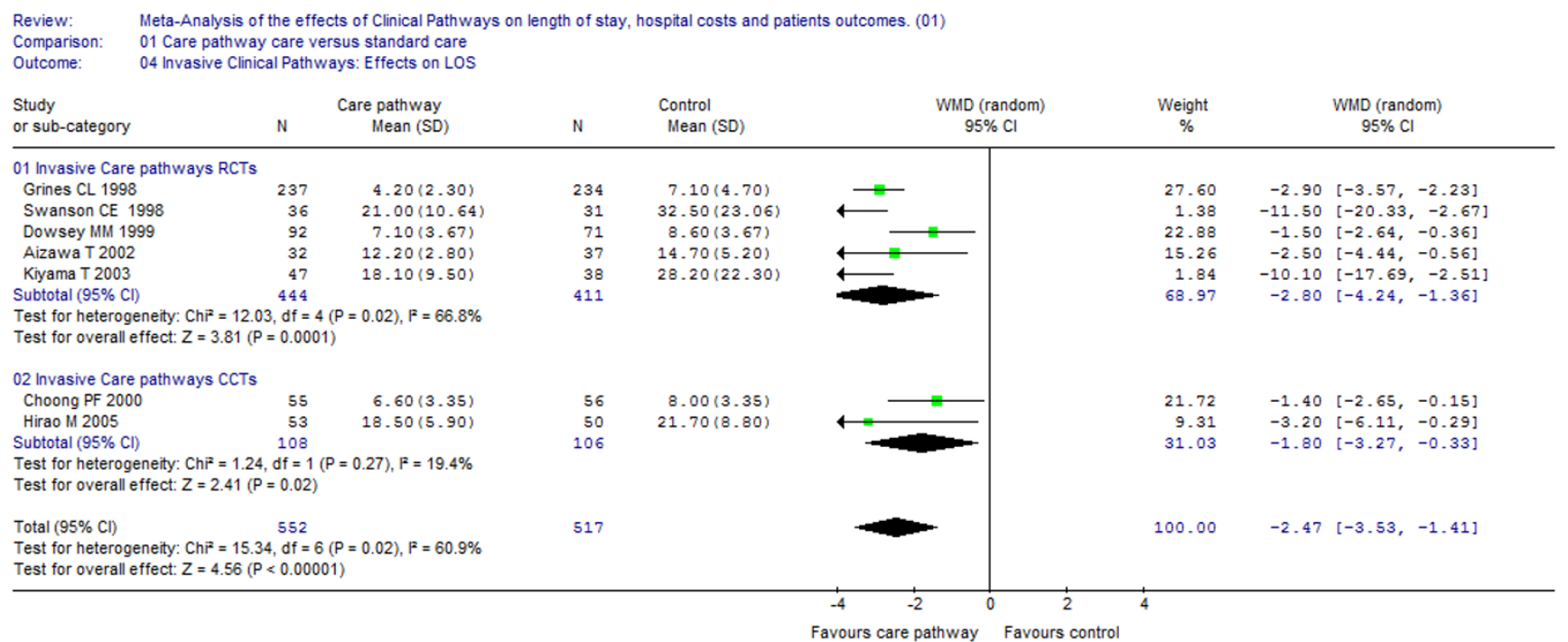

Figure 6

Effects on LOS invasive pathways. [see attached file 6] (BMP Format). 


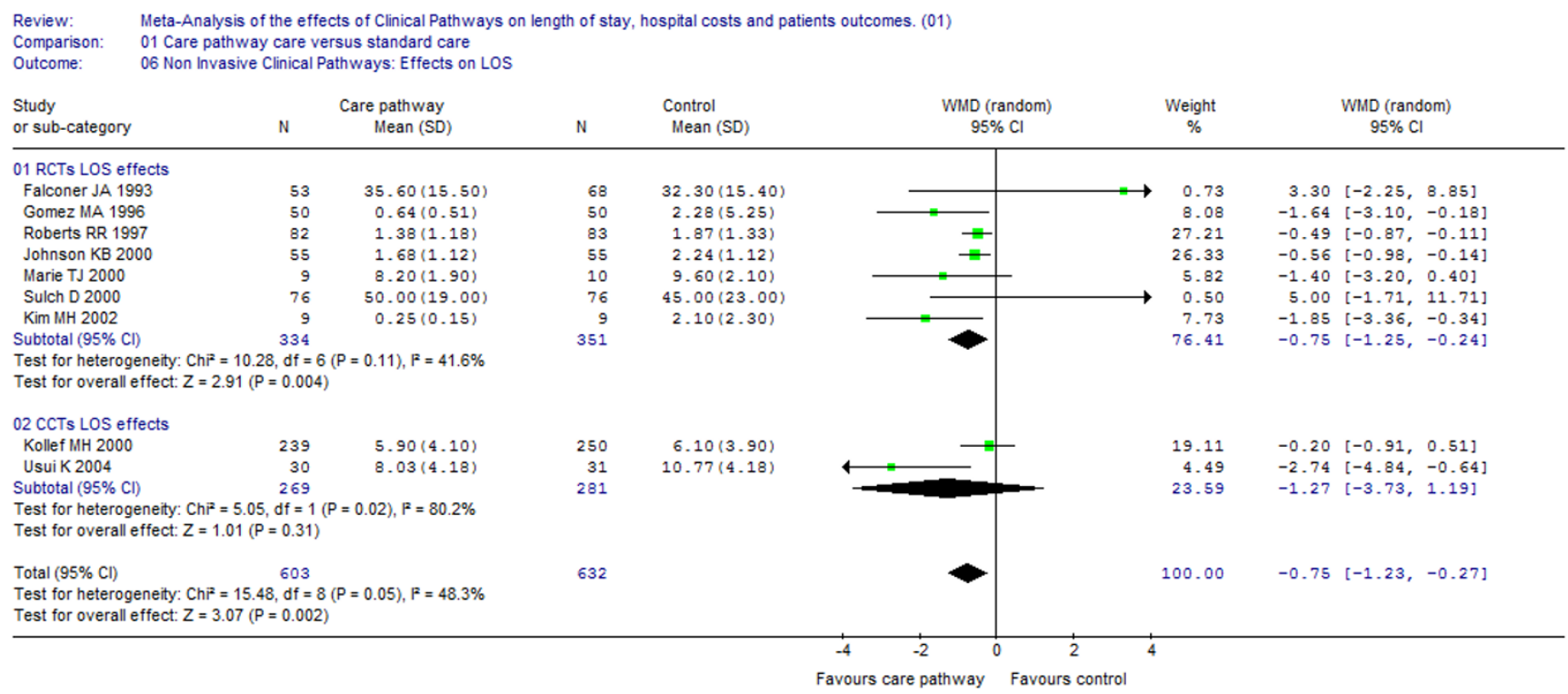

Figure 7

Effects on LOS non-invasive pathways. [see attached file 7] (BMP Format).

thermore, the pooled percentage LOS effects were similar in fixed versus random effects models (WMD - 0.25 versus WMD -0.21).

\section{Discussion}

In general, the majority of studies reporting economic data (LOS and hospital costs) showed a positive impact. The results suggest that clinical pathways for invasive procedures generate clearer LOS effects (WMD -0.8 conservative versus -2.5 invasive).

Clinical pathways appeared to be effective in reducing LOS and costs. Furthermore, pathways for invasive procedures showed a stronger LOS reduction by comparing the magnitudes of effect. These results may not be applied for acute rehabilitation for stroke, where reverse effects were reported (see effects on LOS, Figure 3) [23,38]. Both trends were not statistically significant but they were in contrast to the majority of pathway effects reported in the present review. However, the question of comparability may rise as a reflection of the differing pathway components included in this review and also applies to the kind and number of providers included in the primary studies.

We did not publish our review protocol prior to the study. The review protocol for the follow-up study will be published as a Cochrane review to prevent any doubt about the comparison to be data-driven instead of protocoldriven. We determined the scope of this review question on a pilot analysis of existing primary study data resulting in a diverse set of included studies. As it is an explanatory analysis, the pooled results of the meta-analyses may only apply for the majority of included pathway conditions reporting positive effects or trends. Another limitation refers to the poorly described control conditions reported in the primary studies and implied both, the risk of contamination, and the masking of effects. Therefore, we did not pool the primary LOS and costs data from all of the 17 included studies and concentrated on examining the relationship between clinical subgroups (i.e. surgical versus non-invasive pathway conditions).

It should be noted that the development and implementation of clinical pathways consumes a considerable amount of resources. This corresponds to the fact that truly achievable costs savings depend on the number of cases (volume). This has to be included in the costs analysis. The inflation-adjusted costs for implementation (without maintenance and further development) of the pathway indication "Caesarian section" amounted to nearly $\$ 20,000$ [40]. However, since normally 20 percent of the diagnoses cover 80 percent of the cases [18], a considerable percentage of medical services can be dealt with using a relatively small number of clinical pathways. Therefore, the expenditures will amortize rapidly.

It is very important not to look too far into these results, as there were some limitations. Moreover, it has to be emphasised that evidence determined by meta-analysis is always exploratory in nature and should be considered with caution.

Due to the result of the relatively small number of studies meeting inclusion criteria, this evidence base is not con- 

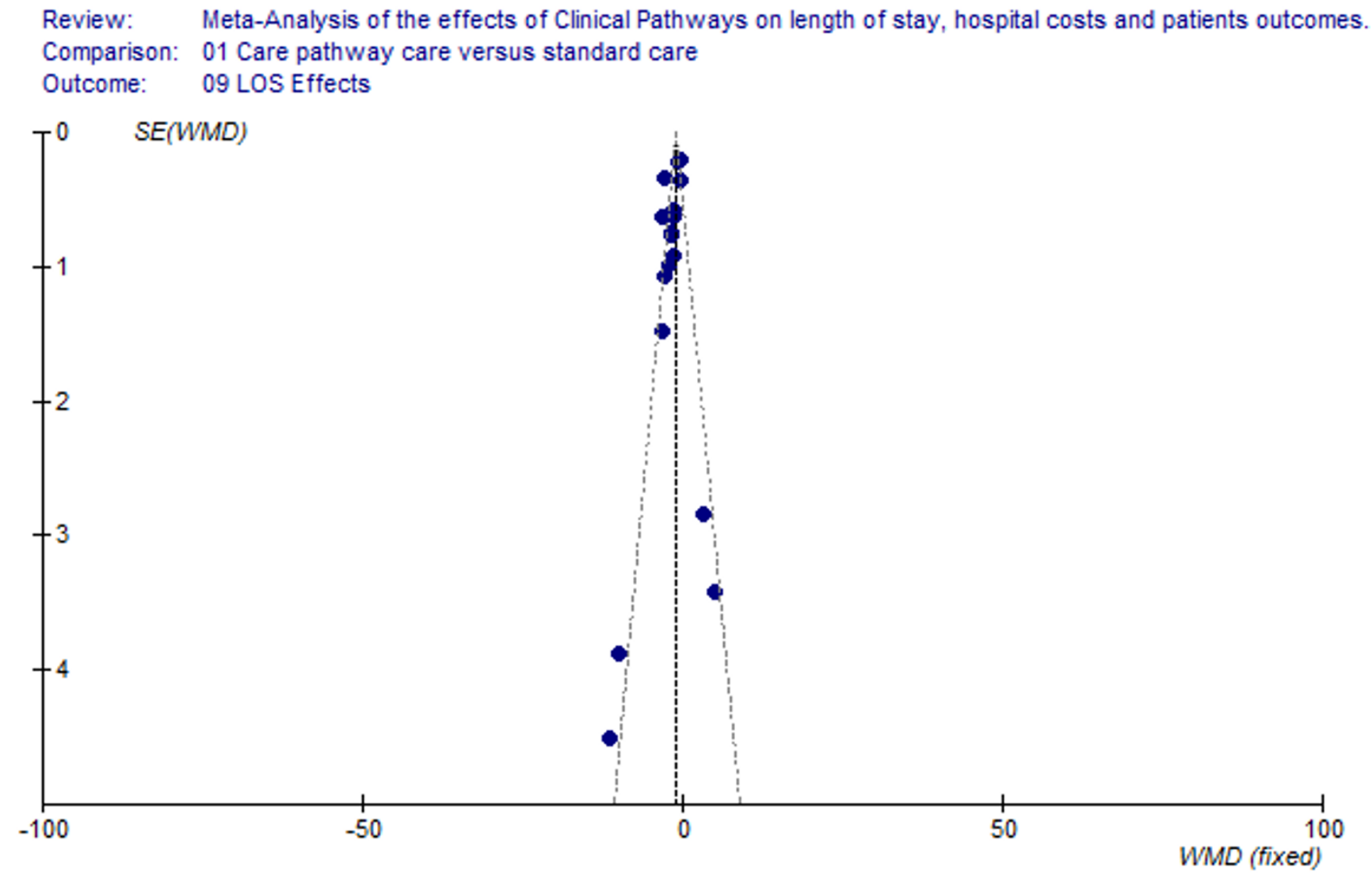

Figure 8

Funnel plot analyses. [see attached file 8] (BMP Format).

clusive enough to provide a replicable framework for all pathway strategies. Considering the clinical areas for implementation, clinical pathways seem to be effective especially for invasive care. The likely benefits and costs need to be considered by the local healthcare providers when implementing clinical pathways under different circumstances. This review has shown that there is not one, singular strong evidence base. Accordingly, decision-makers should also consider some limitations in relation to the generalization of these findings. Replicating the results of this review in other settings could be problematic (e.g. ceiling effects such as market forces).

The heterogeneity in design and outcomes of the studies was large and refers to the statistical heterogeneity in addition to the clinical variability of the included studies. This precluded the overall pooling of LOS and cost data, although the order of magnitude of effects indicated that there are considerable implications of using clinical pathways.
It is unavoidable that some studies will have been overlooked, despite our electronic search strategy. Studies meeting our clinical pathway definition (see Table $1 \& 4$ ) were included, regardless of the fact that the term pathway was mentioned in the study and was done to avoid subjectivity. Also, studies were independently assessed and data extracted by two with any disagreement discussed with a third reviewer.

Finally, should be emphasised that the standard of the primary studies included pose a threat to the validity of the results. While the overall quality of the included studies was moderate, most demonstrated methodological weaknesses such as a small sample size available for analysis.

\section{Conclusion}

With respect to the totality of available evidence, the knowledge about the mechanisms through which pathways work is insufficient. Future research should focus on a better understanding of the key elements of clinical pathways that have impact on economic and patient out- 

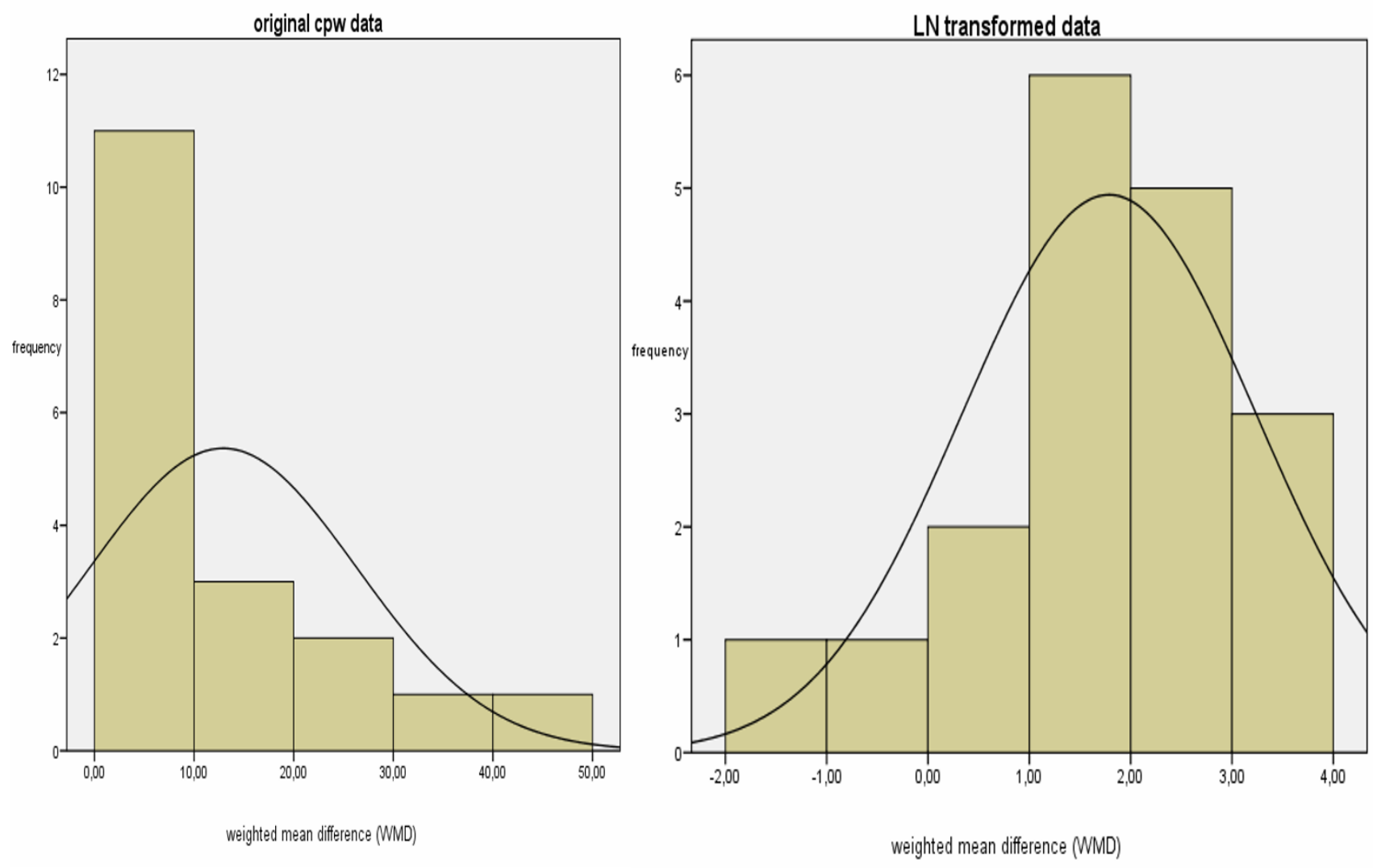

Figure 9

a \& b - Graphic distribution original cpw data and LN transformed. [see attached file 9 a \& b] (PDF Format).

comes. It is also surprising that more studies do not consider any cost effects other than those of treatment. Health-economic research should therefore concentrate on costs of development and implementation of clinical pathways.

This investigation is the first systematic review regarding the effects of clinical pathways on process and patient outcomes. We explicitly decided to expand this review and will also include less restrictive study designs in addition to randomized and quasi-randomized trials, to provide a comprehensive theoretical basis. The character of nonexperimental studies makes them even more difficult to critically assess and moreover, due to the lack of $\mathrm{MeSH}$ terms the search results cannot be as sensitive as those for purely RCT/CCT-based reviews. Another future direction is a more comprehensive, patient-centered approach, concentrating more on patient-outcomes rather then healtheconomic study endpoints. The next scheduled update for this review is planned for the End of 2009.

\section{Competing interests}

The authors declare that they have no competing interests.

\section{Authors' contributions}

TR: Made substantial contributions to conception and design, acquisition of data, analysis and interpretation of data. In particular, he independently screened all titles and abstracts in the first stage of study assessment and led the study assessment of all full text papers as well as the data extraction. He has also been involved in drafting the manuscript and revised it critically as corresponding author. JK: Made substantial contributions to conception and design, acquisition and interpretation of data. He has also been involved in drafting the manuscript, revised it critically for important intellectual content and given final approval. RK: Made substantial contributions to quality assessment, analysis and interpretation of data. In particular, he also independently screened all abstracts, assessed the full text papers and double checked the extracted study data. Has also been involved in drafting the manuscript, revised it critically for statistical content and given final approval. HG: Made substantial contributions to conception and design, acquisition of data and revised the manuscript critically. He has also been involved in developing the electronic-search strategy and any disagreement between TR and RK was discussed with him as a third party reviewer. ST: Has been involved in acquisition of 
data, screening of studies, retrieving potentially relevant studies and has been involved in drafting the manuscript. JMO: Has been involved in screening of studies, retrieving potentially relevant studies, drafting the manuscript and revised it critically for statistical content. EWS: Made substantial contributions to acquisition of data, statistical analysis and interpretation of data. Has also been involved in drafting the manuscript, revised it critically for intellectual content and given final approval.

\section{Additional material}

\section{Additional file 1 \\ Quality criteria for RCTs and CCTs. The depicted quality criteria repre- sent the standard used for quality assessment \\ Click here for file \\ [http://www.biomedcentral.com/content/supplementary/1472- 6963-8-265-S1.pdf]}

\section{Additional file 2}

Formulas log transformation. The depicted formula was used for log transformation

Click here for file

[http://www.biomedcentral.com/content/supplementary/1472-

6963-8-265-S2.pdf]

\section{Additional file 3}

Characteristics of excluded studies. The table briefly reflects the characteristics of excluded studies and the reasons for exclusion

Click here for file

[http://www.biomedcentral.com/content/supplementary/14726963-8-265-S3.pdf]

\section{Additional file 4}

References to excluded studies. The table depicts the references to excluded studies

Click here for file

[http://www.biomedcentral.com/content/supplementary/14726963-8-265-S4.pdf]

\section{Acknowledgements}

This article is based on an oral presentation made at a two day meeting entitled 14th German-Hospital-Controller-Day (14. Deutscher KrankenhausController-Tag) held in Berlin, Germany in March (29th and 30th) 2007.

\section{References}

I. Campbell H, Hotchkiss R, Bradshaw N, Porteous M: Integrated care pathways. $B M]$ 1998, 316:133-137.

2. Coffey RJ, Richards JS, Remmert CS, LeRoy SS, Schoville RR, Baldwin PJ: An introduction to critical paths. Qual Manag Health Care 2005, 14:46-55.

3. Saint S, Hofer TP, Rose JS, Kaufman SR, McMahon LF Jr: Use of critical pathways to improve efficiency: a cautionary tale. Am J Manag Care 2003, 9:758-765.

4. Porter GA, Pisters PW, Mansyur C, Bisanz A, Reyna K, Stanford P, Lee JE, Evans DB: Cost and utilization impact of a clinical pathway for patients undergoing pancreaticoduodenectomy. Annals of Surgical Oncology 2000, 7(7):484-9.
5. Quaglini S, Cavallini A, Gerzeli S, Micieli G: Economic benefit from clinical practice guideline compliance in stroke patient management. Health Policy 2004, 69:305-3 I 5.

6. Roberts HC, Pickering RM, Onslow E, Clancy M, Powell J, Roberts A, Hughes $K$, Coulson D, Bray J: The effectiveness of implementing a care pathway for femoral neck fracture in older people: a prospective controlled before and after study. Age and Ageing 2004, 33: $178-184$.

7. Bailey R, Weingarten S, Lewis M, Mohsenifar Z: Impact of clinical pathways and practice guidelines on the management of acute exacerbations of bronchial asthma. Chest 1998, I I 3:28-33.

8. Kwan J, Sandercock P: In-hospital care pathways for stroke. Cochrane Database Syst Rev 2004:CD002924.

9. Smith TJ, Hillner BE: Ensuring quality cancer care by the use of clinical practice guidelines and critical pathways. J Clin Oncol 200I, I 9:2886-2897.

10. Kim S, Losina E, Solomon DH, Wright J, Katz JN: Effectiveness of clinical pathways for total knee and total hip arthroplasty: literature review. J Arthroplasty 2003, 18:69-74.

II. Banasiak NC, Meadows-Oliver M: Inpatient asthma clinical pathways for the pediatric patient: an integrative review of the literature. Pediatr Nurs 2004, 30:447-450.

12. Higgins JPT, Green S: Cochrane Handbook for Systematic Reviews of Interventions 4.2.5. Chichester, UK: John Wiley \& Sons, Ltd: The Cochrane Library; 2005.

13. Vanhaecht K, De Witte K, Depreitere R, Sermeus W: Clinical pathway audit tools: a systematic review. J Nurs Manag 2006, 14:529-537.

14. MedlinePlus[internet]: (MD)Bethesda: National Library of Medicine (US). [cited 2005 August II]. [http://www.nlm.nih.gov/ medlineplus/mplusdictionary.html].

15. Bero L, Grilli R, Grimshaw JM, Mowat G, Oxman A, Zwarenstein M: Cochrane Effective Practice and Organisation of Care Review Group (Cochrane Group Module). Issue 3 Edited by: Bero L, Grilli R, Grimshaw JM, Mowat G, Oxman A, Zwarenstein M. Oxford: The Cochrane Library; $200 \mathrm{I}$.

16. Drummond MF, Jefferson TO: Guidelines for authors and peer reviewers of economic submissions to the BMJ. The BMJ Economic Evaluation Working Party. BMJ 1996, 3 13:275-283.

17. Review-Manager: (RevMan) [computer program] 4.2 for Windows edition. Copenhagen: The Nordic Cochrane Centre, The Cochrane Collaboration; John Wiley \& Sons, Ltd; 2003.

18. Schlüchtermann J, Sibbel R, Prill MA, Oberender P: Clinical Pathways als Prozesssteuerungsinstrument im Krankenhaus. In Clinical pathways: Facetten eines neuen Versorgungsmodells Edited by: Oberender P. Stuttgart: Kohlhammer Verlag; 2005:43-57.

19. Berry DA: Meta-Analyses in Medicine and Health Policy New York; Basel: Marcel Dekker, Inc; 2000.

20. Soeken KL, Sripusanapan A: Assessing publication bias in metaanalysis. Nurs Res 2003, 52:57-60.

21. Marshall A, Vasilakis C, El-Darzi E: Length of stay-based patient flow models: recent developments and future directions. Health Care Manag Sci 2005, 8:213-220.

22. SPSS: Statistical Product and Service Solutions (SPSS) for Windows. Version I5.0 edition. Chicago, IL 2006.

23. Falconer JA, Roth EJ, Sutin JA, Strasser DC, Chang RW: The critical path method in stroke rehabilitation: lessons from an experiment in cost containment and outcome improvement. $Q R B$ Qual Rev Bull 1993, 19(1):8-16.

24. Swanson CE, Day GA, Yelland CE, Broome JR, Massey L, Richardson HR, Dimitri K, Marsh A: The management of elderly patients with femoral fractures. A randomised controlled trial of early intervention versus standard care. Med J Aust 1998, 169:515-518.

25. Chen SH, Yeh KW, Chen SH, Yen DC, Yin TJ, Huang JL: The development and establishment of a care map in children with asthma in Taiwan. J Asthma 2004, 41:855-86I.

26. Grines CL, Marsalese DL, Brodie B, Griffin J, Donohue B, Costantini CR, Balestrini C, Stone G, Wharton T, Esente P, Spain M, Moses J, Nobuyoshi M, Ayres M, Jones D, Mason D, Sachs D, Grines LL, O'Neill W: Safety and cost-effectiveness of early discharge after primary angioplasty in low risk patients with acute myocardial infarction. PAMI-II Investigators. Primary Angioplasty in Myocardial Infarction. J Am Coll Cardiol 1998, 31:967-972 
27. Dowsey MM, Kilgour ML, Santamaria NM, Choong PF: Clinical pathways in hip and knee arthroplasty: a prospective randomised controlled study. Med J Aust 1999, I 70:59-62.

28. Gomez MA, Anderson JL, Karagounis LA, Muhlestein JB, Mooers FB: An emergency department-based protocol for rapidly ruling out myocardial ischemia reduces hospital time and expense: results of a randomized study (ROMIO). J Am Coll Cardiol 1996, 28:25-33.

29. Aizawa T, Kin T, Kitsukawa S, Mamiya Y, Akiyama A, Ohno Y, Okubo $Y$, Miki M, Tachibana M: [Impact of a clinical pathway in cases of transurethral resection of the prostate]. Nippon Hinyokika Gakkai Zasshi 2002, 93:463.

30. Marrie TJ, Lau CY, Wheeler SL, Wong CJ, Vandervoort MK, Feagan BG: A controlled trial of a critical pathway for treatment of community-acquired pneumonia. CAPITAL Study Investigators. Community-Acquired Pneumonia Intervention Trial Assessing Levofloxacin. JAMA 2000, 283:749-755.

31. Roberts RR, Zalenski RJ, Mensah EK, Rydman RJ, Ciavarella G, Gussow L, Das K, Kampe LM, Dickover B, McDermott MF, Hart A, Straus HE, Murphy DG, Rao R: Costs of an emergency departmentbased accelerated diagnostic protocol vs hospitalization in patients with chest pain: a randomized controlled trial. JAMA 1997, 278:1670-1676.

32. Johnson KB, Blaisdell CJ, Walker A, Eggleston P: Effectiveness of a clinical pathway for inpatient asthma management. Pediatrics 2000, 106:1006-1012.

33. Kim MH, Morady F, Conlon B, Kronick S, Lowell M, Bruckman D, Armstrong WF, Eagle KA: A prospective, randomized, controlled trial of an emergency department-based atrial fibrillation treatment strategy with low-molecular-weight heparin (Structured abstract). Ann Emerg Med 2002, 40:187-192.

34. Kollef MH, Shapiro SD, Clinkscale D, Cracchiolo L, Clayton D, Wilner $R$, Hossin $L$ : The effect of respiratory therapist-initiated treatment protocols on patient outcomes and resource utilization. Chest 2000, I I 7:467-475.

35. Choong PF, Langford AK, Dowsey MM, Santamaria NM: Clinical pathway for fractured neck of femur: a prospective, controlled study. Med J Aust 2000, I 72:423-426.

36. Usui K, Kage H, Soda M, Noda H, Ishihara T: Electronic clinical pathway for community acquired pneumonia (e-CP CAP) (Structured abstract). Nihon Kokyuki Gakkai Zasshi 2004, 42:620-624.

37. Hirao M, Tsujinaka T, Takeno A, Fujitani K, Kurata M: Patient-controlled dietary schedule improves clinical outcome after gastrectomy for gastric cancer. World J Surg 2005, 29:853-857.

38. Sulch D, Perez I, Melbourn A, Kalra L: Randomized controlled trial of integrated (managed) care pathway for stroke rehabilitation. Stroke 2000, 31:1929-1934.

39. Kiyama T, Tajiri T, Yoshiyuki T, Mitsuhashi K, Ise Y, Mizutani T, Okuda T, Fujita I, Masuda G, Kato S, Matsukura N, Tokunaga A, Hasegawa S: Clinical significance of a standardized clinical pathway in gastrectomy patients (Structured abstract). J Nippon Med Sch 2003, 70:263-269.

40. Comried LA: Cost analysis: initiation of HBMC and first CareMap. Nurs Econ 1996, 14:34-39.

\section{Pre-publication history}

The pre-publication history for this paper can be accessed here:

http://www.biomedcentral.com/1472-6963/8/265/pre pub

\section{Publish with Biomed Central and every} scientist can read your work free of charge

"BioMed Central will be the most significant development for disseminating the results of biomedical research in our lifetime. "

Sir Paul Nurse, Cancer Research UK

Your research papers will be:

- available free of charge to the entire biomedical community

- peer reviewed and published immediately upon acceptance

- cited in PubMed and archived on PubMed Central

- yours - you keep the copyright
BioMedcentral 